\section{THE BIOAVAILABILITY AND MATURING CLEARANCE OF DOXAPRAM IN PRETERM INFANTS}

${ }^{1,2}{ }^{2} \mathrm{RB}$ Flint*, ${ }^{1}$ SHP Simons, ${ }^{3} \mathrm{P}$ Andriessen, ${ }^{4} \mathrm{KD}$ Liem, ${ }^{5} \mathrm{PL}$ Degraeuwe, ${ }^{11} \mathrm{IKM}$ Reiss, ${ }^{6} \mathrm{R}$ Ter Heine, ${ }^{7} \mathrm{AGJ}$ Engbers, ${ }^{2} \mathrm{BCP}$ Koch, ${ }^{8} \mathrm{R}$ De Groot, ${ }^{6} \mathrm{DM}$ Burger, ${ }^{1,7,9} \mathrm{CAJ}$ Knibbe, ${ }^{7} \mathrm{~S}$ Völler, DINOResearch Group. 'Department of Pediatrics, Division of Neonatology, Erasmus MC Sophia Children's Hospital; ${ }^{2}$ Department of Pharmacy, Erasmus MC, Rotterdam; ${ }^{3}$ Maxima Medical Center, Veldhoven; ${ }^{4}$ Department of Neonatology, Radboud UMC-Amalia Children's Hospital, Nijmegen; ${ }^{5}$ Department of Neonatology, Maastricht University Medical Center, Maastricht; ${ }^{6}$ Department of Pharmacy and Radboud Institute of Health Sciences (RIHS), Radboud University Medical Center, Nijmegen; ' Division of Pharmacology, Leiden Academic Centre for Drug Research, Leiden University, Leiden; ${ }^{8}$ Laboratory of Pediatric Infectious Diseases, Department of Pediatrics, Radboud Institute for Molecular Life Sciences, Radboud University Medical Center, Nijmegen; ${ }^{9}$ Department of Clinical Pharmacy, St Antonius Hospital, Nieuwegein, The Netherlands

\subsection{6/archdischild-2019-esdppp.17}

Background Doxapram is used for intravenous and oral treatment of apnea of prematurity in preterm infants. Dosing is currently based on bodyweight, however pharmacokinetic and bioavailability data are limited. To develop individualized dosing strategies, we characterized pharmacokinetics of doxapram in this vulnerable patient population.

Methods Data (302 samples) from 75 neonates were included with median (range) gestational age (GA) 25.9 (23.9-29.4) weeks, bodyweight $0.95(0.48-1.61) \mathrm{kg}$, postnatal age (PNA) 17 (1-52) days at start of continuous treatment, and treatment duration of 16.8 (1.4-26.9) days. A population pharmacokinetic model was developed for doxapram and keto-doxapram. Results A two-compartment model best described the pharmacokinetics of doxapram and its metabolite. PNA and GA affected the formation clearance of keto-doxapram $\left(\mathrm{CL}_{\mathrm{D}-\mathrm{KD}}\right)$ and clearance of doxapram via other routes $\left(\mathrm{CL}_{\mathrm{D}}\right)$. For an individual of $0.95 \mathrm{~kg}$, GA of 25.9 weeks and PNA of 17 days, $\mathrm{CL}_{\mathrm{D}-\mathrm{KD}}$ was $0.096 \mathrm{~L} / \mathrm{h}$ (residual standard error (RSE) $22 \%$ ) and $\mathrm{CL}_{\mathrm{D}} 0.493 \mathrm{~L} / \mathrm{h}$ (RSE 13\%). Compared to PNA 30 days, estimated $\mathrm{CL}_{\mathrm{D}-\mathrm{KD}}$ was $13 \%$ at PNA day 1 and $69 \%$ at day 15 , and estimated $C_{D}$ was $12 \%$ and $68 \%$, respectively. Compared to GA 28 weeks, estimated $\mathrm{CL}_{\mathrm{D}-\mathrm{KD}}$ was $65 \%$ at GA 24 weeks and $80 \%$ at 26 weeks, and estimated $\mathrm{CL}_{\mathrm{D}}$ was $67 \%$ and $81 \%$, respectively. Oral bioavailability was estimated 74\% (RSE 13\%).

Conclusions With bodyweight-based dosing alone, preterm infants with the lowest PNA and GA had relatively low doxapram CL and the highest exposure. Therefore, the dose may be reduced by $50 \%$ up to PNA day 9 , and by $25 \%$ for day 10-15. In addition, for GA dose may be reduced by $40 \%$ and $20 \%$ in newborns with GA of 24-25 weeks and 26-27 weeks, respectively, compared to $28-29$ weeks. For switch to oral therapy a $33 \%$ dose increase is required to maintain plasma concentrations.

Disclosure(s) Nothing to disclose

\section{THERAPEUTIC DELIVERY DURING BREASTFEEDING: A FEASIBILITY STUDY}

${ }^{1,2} \mathrm{~T}_{\text {Maier }}{ }^{*},{ }^{3} \mathrm{P}$ Peirce, ${ }^{3} \mathrm{~L}$ Baird, ${ }^{4} \mathrm{SL}$ Whitehouse, ${ }^{2} \mathrm{NKH}$ Slater, ${ }^{1,3} \mathrm{~K}$ Beardsall. ${ }^{1}$ Department of Paediatrics; ${ }^{2}$ Department of Chemical Engineering and Biotechnology, University of Cambridge; ${ }^{3}$ Cambridge University Hospitals NHS Foundation Trust, Cambridge; ${ }^{4}$ College of Social Sciences, Arts and Humanities, University of Leicester, Leicester, UK

10.1136/archdischild-2019-esdppp.18

Background At an age when breastfeeding is the optimal nutritional support for infants, enteral drug delivery can be physically and emotionally challenging for parents. Delivery during breastfeeding could serve as an alternative to currently existing approaches. This study aimed to explore its feasibility and acceptability.

Methods Vitamin B12 was administered as part of a singlecentre feasibility study to breastfed infants at the University of Cambridge Addenbrooke's Hospital NHS Trust. Hereby a solid formulation (tablet) was placed inside an ultra-thin silicone nipple shield, and worn by a mother during the feed. The study investigated i.) quantitative changes in B12 blood serum levels at baseline and 6-8 hours after the study feed, ii.) mothers' expectations and experiences via a mixed method approach by a single investigator. Local ethics approval was obtained prior to any study procedures being undertaken (18/ LO/0551).

Results Twenty dyads completed the study protocol. In all cases, no residual tablet was left after the feed, and the tablet's presence within the shield did not appear to impact feeding. A pharmacokinetic-dependent vitamin B12 increase to $1871 \mathrm{pg} / \mathrm{mL}(610-4981 \mathrm{pg} / \mathrm{mL})$ from a baseline of $533 \mathrm{pg} / \mathrm{mL}$ (236-925 pg/mL) was observed. Mothers described the nipple shield's surprising ease of use and comfort for delivery, not affecting normal breastfeeding behaviour/sensation, while decreasing infant/maternal distress compared to the use of an oral syringe. All mothers expressed their wish for this approach to become available to parents in the future. Reasoning included the desire (1) of parents to have choices in relation to their infants' health, (2) to replace a medical intervention with one that was felt to be more 'natural'.

Conclusions This study showed that solid formulations can be used for therapeutic delivery whilst breastfeeding and is viewed by mothers as advantageous compared to currently available methods.

Disclosure(s)

Funding The research was supported by a WD Armstrong $\mathrm{PhD}$ studentship for the Application of Engineering in Medicine, University of Cambridge, the German Academic Scholarship Foundation, and the Kurt Hahn Trust, University of Cambridge. Competing interests (applicable to all authors): None declared.

\section{DEVELOPMENT OF A PAEDIATRIC BRAIN PBPK MODEL IN CHILDREN WITH AND WITHOUT MENINGITIS}

${ }^{1}$ LFM Verscheijden*, ${ }^{1} \mathrm{JB}$ Koenderink, ${ }^{2,3} \mathrm{~K}$ Allegaert, ${ }^{1,2} \mathrm{SN}$ de Wildt, ${ }^{1} \mathrm{FGM}$ Russel. ${ }^{1}$ Department of Pharmacology and Toxicology, Radboud Institute for Molecular Life Sciences, Nijmegen; ${ }^{2}$ Intensive Care and Department of Paediatric Surgery, Erasmus MC Sophia Childrens Hospital, Rotterdam, The Netherlands; ${ }^{3}$ Department of Development and Regeneration, KU Leuven, Leuven, Belgium

\subsection{6/archdischild-2019-esdppp. 19}

Background Several paediatric physiologically-based pharmacokinetic (PBPK) models have been developed that incorporate developmental changes affecting plasma drug concentrations. Disposition into cerebrospinal fluid (CSF) is also age-related and influenced by physiological factors, including CSF production rate, but also by brain diseases, such as meningitis, which are associated with impaired blood-brain barrier integrity. Our aim was to develop a paediatric brain PBPK model to predict CSF drug concentrations in children with and without meningitis.

Methods A paediatric PBPK model was developed incorporating age-appropriate parameters and associated inter-individual 
variability. The model was validated for paracetamol, ibuprofen, flurbiprofen and naproxen, and for a paediatric meningitis population by estimating meropenem blood-brain barrier penetration using sensitivity analysis. Plasma and CSF drug concentrations derived from literature were used to perform visual predictive checks and to calculate ratios between simulated and observed AUCs in order to evaluate model performance. Results Simulated data were comparable to observed over a broad age range (1 day - 15 y postnatal age), for all drugs investigated. The ratios between observed and simulated AUCs were within 2-fold difference both in plasma and in CSF, indicating acceptable model performance. Disposition of meropenem into the brain was slow and CSF concentrations were lower compared to plasma concentrations. In addition, several days were needed to achieve CSF steady-state concentration.

Conclusions Our paediatric brain PBPK model provides a new tool to predict CSF concentrations in children with and without meningitis and can be used as a template model for other drugs acting in the CNS.

Disclosure(s) Nothing to disclose

\section{O20 DOSE-LINEARITY OF THE PHARMACOKINETICS OF AN INTRAVENOUS $\left[{ }^{14} \mathrm{C}\right]$ MIDAZOLAM MICRODOSE IN CHILDREN}

${ }^{1} \mathrm{BD}$ van Groen*, ${ }^{2}$ WHJ Vaes, ${ }^{3} \mathrm{BK}$ Park, ${ }^{4} \mathrm{EHJ}$ Krekels, ${ }^{2} \mathrm{E}$ van Duijn, ${ }^{5} \mathrm{~L}-\mathrm{T}$ Kõrgvee, ${ }^{6} \mathrm{~W}$ Maruszak, ${ }^{6} \mathrm{G}$ Grynkiewicz, ${ }^{7} \mathrm{RC}$ Garner, ${ }^{4,8} \mathrm{C}$ Knibbe, ${ }^{1} \mathrm{D}$ Tibboel, ${ }^{1,9} \mathrm{SN}$ de Wildt*, ${ }^{3}$ MA Turner*. 'Erasmus MC - Sophia Children's Hospital, Rotterdam; ${ }^{2}$ TNO, Zeist, The Netherlands; ${ }^{3}$ University of Liverpool, Liverpool, UK; ${ }^{4}$ Leiden University, Leiden, The Netherlands; ${ }^{5}$ University of Tartu, Tartu, Estonia; ${ }^{6}$ Pharmaceutical Research Institute, Warsaw, Poland; ' Garner Consulting, York, UK; ${ }^{8}$ St. Antonius Hospital, Nieuwegein; ${ }^{9}$ Radboudumc, Nijmegen, The Netherlands

\subsection{6/archdischild-2019-esdppp.20}

Background Drug disposition in children may vary from adults due to age-related variation in drug metabolism, but paediatric pharmacokinetic (PK) studies are challenging. Microdose studies present an innovation to study $\mathrm{PK}$ in paediatrics, and can only be used when the PK of a microdose are dose-linear to a therapeutic dose. We aimed to assess dose-linearity of $\left[{ }^{14} \mathrm{C}\right]$ midazolam (MDZ), a marker for the activity of the developmentally regulated CYP3A enzyme, by comparing the PK of an intravenous (IV) $\left[{ }^{14} \mathrm{C}\right] \mathrm{MDZ}$ microtracer given simultaneously with therapeutic MDZ, with the PK of a single IV $\left[{ }^{14} \mathrm{C}\right]$ MDZ microdose.

Methods Preterm to 2-year-old infants admitted to the intensive care unit received $\left[{ }^{14} \mathrm{C}\right] \mathrm{MDZ}$ IV either as a microtracer during therapeutic MDZ infusion or as an isolated microdose. Dense blood sampling was done up to 36 hours after dosing. Plasma concentrations of $\left[{ }^{14} \mathrm{C}\right] \mathrm{MDZ}$ and $\left[{ }^{14} \mathrm{C}\right] 1-\mathrm{OH}-\mathrm{MDZ}$ were determined by accelerator mass spectrometry. A population PK model was developed with NONMEM 7.4 to study whether there was a difference in the PK of the microtracer versus those of a microdose $\left[{ }^{14} \mathrm{C}\right] \mathrm{MDZ}$.

Results Of fifteen children (median gestational age 39.4 [range 23.9-41.4] weeks, postnatal age 11.4 [0.6-49.1] weeks), nine received a microdose and six a microtracer $\left[{ }^{14} \mathrm{C}\right] \mathrm{MDZ}(111$ $\mathrm{Bq} / \mathrm{kg} ; 37.6 \mathrm{ng} / \mathrm{kg})$. In a two-compartment PK model, bodyweight was the most significant covariate for volume of distribution. There was no statistically significant difference in any PK parameter between the $\left[{ }^{14} \mathrm{C}\right] \mathrm{MDZ}$ microdose or microtracer, suggesting the PK of MDZ to be linear within the range of the therapeutic doses and microdoses.

Conclusion Our data supports the dose-linearity of an IV $\left[{ }^{14} \mathrm{C}\right] \mathrm{MDZ}$ microdose in children, thus a $\left[{ }^{14} \mathrm{C}\right] \mathrm{MDZ}$ microdosing approach can be used to study developmental changes in hepatic CYP3A activity.

Disclosure(s) This project was funded by the ZonMw ERANET PRIOMEDCHILD programme (projectnumber 113205022). * both authors contributed equally

\section{PROGRESS TOWARDS THERAPEUTIC DRUG MONITORING VIA BREATH ANALYSIS}

KD Singh, V Ziesenitz, J Usemann, J Van Den Anker, U Frey, N von der Weid, A Datta, P Sinues*. University Children's Hospital Basel (UKBB), University of Basel, Basel, Switzerland

\subsection{6/archdischild-2019-esdppp.21}

Background Therapeutic Drug Monitoring (TDM) is essential aspect for the clinical management of patients. However, despite the clear advantages of TDM, it faces several challenges to being more widely used in the clinic. Specially challenging is the case of TDM in children, as they experience rapid physiologic developments, leading to great pharmacokinetic and pharmacodynamic variability. Breath analysis provides a patient-friendly approach to support TDM. To explore this possibility, we are currently running a pilot study, whereby we analyze the exhaled breath of pediatric patients receiving anti-seizure or chemotherapy drugs requiring TDM.

Methods We analyzed by secondary electrospray ionizationhigh resolution mass spectrometry (SESI-HRMS) exhaled breath of pediatric patients under therapy for Valproic acid (VPA; $\mathrm{n}=27)$, Lamotrigine $(\mathrm{n}=19)$, Levetiracetam $(\mathrm{n}=$ 15), Oxcarbazepine $(n=11)$ and Methotrexate $(n=4)$. Systemic blood concentrations were measured simultaneously to the breath test. In the case of VPA, we constructed a regression model to predict systemic blood concentrations based on the signal intensity of breath mass spectral features. For the rest of the drugs listed, due to its current limited size, we conducted preliminary data visualization approaches.

Results We found that exhaled metabolites of VPA allow to predict free VPA blood concentrations with a root-meansquare error of $1.5 \mathrm{mg} / \mathrm{L}$ for concentrations in the range $0-12$ $\mathrm{mg} / \mathrm{L}$. This prediction is accomplished within 20 minutes, comprising the breath test and data analysis. For Levetiracetam, Lamotrigine and Oxcarbazepine the data analysis is still ongoing. For MTX we found breath metabolites clearly altered as a result of the drug administration. However, a great inter-individual variability was also observed.

Conclusion We conclude that breath analysis may support current TDM approaches. This will lead to new opportunities to guide the dose of drugs with a high level of accuracy, in realtime and non-invasively.

Disclosure(s) PS gratefully acknowledges the financial support of the Fondation Botnar (Switzerland) and the Swiss National Science Foundation (320030_173168 and PCEGP3_181300). PS is part of the board of directors of Deep Breath Initiative AG. JU was supported by research fellowship of the University Children's Hospital Basel. VS and JVDA were supported by the Eckenstein-Geigy Foundation. "AD and PS: shared senior authorship 